EUROPEAN JOURNAL OF PURE AND APPLIED MATHEMATICS

Vol. 13, No. 3, 2020, 674-696

ISSN 1307-5543 - www.ejpam.com

Published by New York Business Global

\title{
Efficient Zero Ring Labeling of Graphs
}

\author{
Dhenmar E. Chua ${ }^{1,2, *}$, Francis Joseph H. Campeña ${ }^{1}$, Floresto A. Franco Jr. ${ }^{1,3}$ \\ 1 Mathematics and Statistics Department, De La Salle University, Manila, Philippines \\ 2 Mathematics and Physics Department, Adamson University, Manila, Philippines \\ ${ }^{3}$ Mathematics Department, Mariano Marcos State University, Batac, Ilocos Norte, \\ Philippines
}

\begin{abstract}
A zero ring is a ring in which the product of any two elements is zero, which is the additive identity. A zero ring labeling of a graph is an assignment of distinct elements of a zero ring to the vertices of the graph such that the sum of the labels of any two adjacent vertices is not the zero element in the ring. Given a zero ring labeling of a graph, if the cardinality of the set of distinct sums obtained from all adjacent vertices is equal to the maximum degree of the graph, then the zero ring labeling is efficient. In this paper, we showed the existence of an efficient zero ring labeling for some classes of trees and their disjoint union. In particular, we showed that an efficient zero ring labeling exists for some families of the following classes of trees: path graphs, star graphs, bistars, centipede graphs, caterpillars, spiders, lobsters, and rooted trees. We also showed results for other common classes of graphs.
\end{abstract}

2020 Mathematics Subject Classifications: 05C05, 05C25, $05 \mathrm{C} 78$

Key Words and Phrases: Efficient zero ring labeling, zero ring labeling, zero ring

\section{Introduction}

Graph labeling is an assignment of labels to vertices or edges of a graph. Interesting questions naturally arise from this topic, so there has been a vast amount of literature that aimed to answer these questions while consequently introducing new problems.

In 2014, Acharya et al [1] introduced zero ring labeling. In this labeling, each vertex is assigned a unique label from a zero ring such that the sum of any two adjacent vertices is not zero, i.e., the additive identity of the zero ring. It was proved that every graph admits a zero ring labeling with respect to some zero ring.

The zero ring index of a graph, which is the smallest order of a zero ring in which the graph admits a zero ring labeling, was also studied for some well-known graphs. Pranjali et al [6] determined a necessary and sufficient condition for a finite graph of order $n$ to attain an optimal zero ring index of $n$.

*Corresponding author.

DOI: https://doi.org/10.29020/nybg.ejpam.v13i3.3780

Email addresses: chuadhenmar@gmail.com (D. Chua),

francis.campena@dlsu.edu.ph (F. Campeña), otserolf@yahoo.com (F. Franco) 
Dela Rosa-Reynera [2] constructed optimal zero ring labelings for some classes of graphs. Included are labeling schemes for trees and cactus graphs. Moreover, the zero ring indices of graphs that result from some graph operations were also determined.

In this paper, we extend the notion of zero ring labeling further. Can a zero ring labeling be constructed for a graph such that the number of distinct sums is equal to some number? In particular, we want to determine a zero ring labeling where this number is as small as possible.

\section{Preliminaries}

\subsection{Basic definitions}

We now present some definitions used in this paper. For graph theory, [3, 5] are considered, while [4] is used for abstract algebra.

Definition 1. A graph $G=(V, E)$ is a pair of sets $V$ and $E$ such that $E$ is a set of two-element subsets of $V$. The elements of $V$ are the vertices of $G$, while the elements of $E$ are the edges of $G$.

The vertex set of a graph $G$ is denoted by $V(G)$, while its edge set is denoted by $E(G)$. We say that $e$ is an edge at $v$ if $v \in e$. An edge $\{u, v\}$ can be written as $u v$; we say that $u v$ joins $u$ and $v$, and $u$ and $v$ are the ends of $u v$. The number of vertices of a graph $G$ is its order, denoted by $|G|$.

The degree of a vertex $v$, denoted by $d(v)$, is the number of edges at $v$. Two vertices $u$ and $v$ in a graph $G$ are adjacent if $u v$ is an edge in $G$.

A graph $G$ of order $n$ is a complete graph, denoted by $K_{n}$, if for any two distinct vertices $u$ and $v$ in $G, u v$ is an edge in $G$.

Definition 2. The maximum degree of a graph $G$, denoted by $\Delta(G)$, is the highest degree of a vertex in $G$.

Definition 3. A path in a graph is a subgraph $P=\left[v_{1}, v_{2}, \ldots, v_{n}\right]$ such that $V(P)=$ $\left\{v_{1}, v_{2}, \ldots, v_{n}\right\}$ and $E(P)=\left\{v_{1} v_{2}, v_{2} v_{3}, \ldots, v_{n-1} v_{n}\right\}$, where $v_{1}, v_{2}, \ldots, v_{n}$ are distinct.

The number of edges in a path is its length. In a path $P=\left[v_{1}, v_{2}, \ldots, v_{n}\right]$, the vertices $v_{1}$ and $v_{n}$ are the endvertices, while $v_{2}, v_{3}, \ldots, v_{n-1}$ are the inner vertices. Moreover, we say that $P$ is a path between $v_{1}$ and $v_{n}$. The distance between two vertices $u$ and $v$ in a graph $G$ is the length of the shortest path between $u$ and $v$.

Definition 4. A graph $G$ is connected if for any vertices $u$ and $v$ in $G$, there is a path between $u$ and $v$.

A connected subgraph of a graph $G$ is a component of $G$ if it is not a proper subgraph of any other connected subgraph in $G$. 


\subsection{Trees}

Definition 5. A tree is a connected graph with no cycle. The vertices of degree one in a tree are its leaves.

Definition 6. A path graph $P_{n}$, where $n \geq 2$, is a tree with two leaves and $n-2$ vertices with degree two.

Definition 7. A star graph $S_{n}$, where $n \geq 1$, is a tree with one vertex of degree $n-1$ and all others with degree one.

The vertex with the maximum degree in a star graph is its center.

Definition 8. A bistar $B_{n}$, where $n \geq 1$, is a graph formed by joining the centers of two star graphs $S_{n}$.

Definition 9. An $\boldsymbol{n}$-centipede, where $n \geq 1$, is a tree with vertex set $A \cup B$, where $A=\left\{a_{1}, a_{2}, \ldots, a_{n}\right\}$ and $B=\left\{b_{1}, b_{2}, \ldots, b_{n}\right\}$, and edge set $E=\left\{a_{i} b_{i}: 1 \leq i \leq n\right\} \cup$ $\left\{a_{i} a_{i+1}: 1 \leq i \leq n-1\right\}$.

Definition 10. A caterpillar is a tree in which all vertices are within distance one of a central path.

We define a hanging leaf of a vertex in the central path as a leaf that is adjacent to it but not a vertex in the central path.

Example 1. The graph in Fig. 1 is a caterpillar with respect to any of the following central paths: $[c, d, e, f],[a, c, d, e, f],[g, c, d, e, f],[c, d, e, f, j],[a, c, d, e, f, j]$, or $[g, c, d, e, f, j]$. The hanging leaves of $d$ are $i, b$, and $h$.

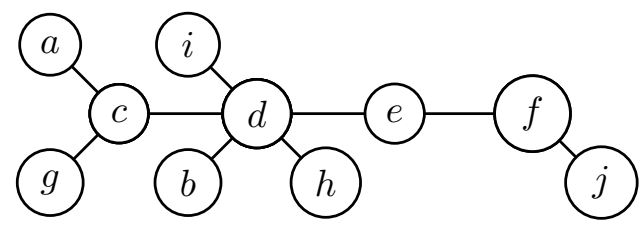

Figure 1: A caterpillar

Definition 11. A spider is a tree with a unique vertex of degree at least three and all others with degree at most two.

We define the head of a spider as the vertex with the maximum degree and a leg as a component of the graph that is obtained by removing the head; that is, a leg is a path.

Definition 12. A lobster is a tree in which all vertices are within distance two of a central path. 
We define an elbow of a vertex in the central path of a lobster as a vertex that is adjacent to it and whose distance from the central path is one. Moreover, we define a hanging leaf of an elbow as a vertex that is adjacent to it and whose distance from the central path is two.

Definition 13. A rooted tree is a tree in which a particular vertex is designated as the root.

The level of a vertex in a rooted tree is its distance from the root, and the height of a rooted tree is the maximum level of a vertex. A child (plural: children) of a vertex of level $n$ in a rooted tree is an adjacent vertex whose level is $n+1$.

\subsection{Common classes of graphs}

We now define other common classes of graphs that are considered in this paper.

Definition 14. A cycle graph $C_{n}=\left[v_{1}, v_{2}, \ldots, v_{n}, v_{1}\right]$ is a graph with vertex set $V=$ $\left\{v_{1}, v_{2}, \ldots, v_{n}\right\}$ and edge set $E=\left\{v_{1} v_{2}, v_{2} v_{3}, \ldots, v_{n-1} v_{n}\right\} \cup\left\{v_{1} v_{n}\right\}$, where $n \geq 3$.

Definition 15. A complete bipartite graph $K_{m, n}$ is a graph with vertex set $V=$ $\left\{x_{1}, x_{2}, \ldots, x_{m}\right\} \cup\left\{y_{1}, y_{2}, \ldots, y_{n}\right\}$, where $m \geq 1$ and $n \geq 1$, and edge set $E=\left\{x_{i} y_{j}: 1 \leq\right.$ $i \leq m$ and $1 \leq j \leq n\}$.

Definition 16. A fan graph $F_{n}$ is a graph that is obtained by joining all vertices of $P_{n}$ to another vertex.

Definition 17. A wheel graph $W_{n}$ is a graph that is obtained by joining all vertices of $C_{n}$ to another vertex.

Definition 18. A friendship graph $T_{n}$ is a graph that consists of $n$ copies of $C_{3}$ having exactly one common vertex.

\subsection{Efficient zero ring labeling}

We now define a vertex labeling of a graph which was introduced by Acharya et al $[1,6]$ called zero ring labeling. Moreover, this paper introduces a variation of this vertex labeling called $k$-zero ring labeling and efficient zero ring labeling of graphs. In this study, the zero ring that will be used in the vertex labelings is the zero ring $M_{2}^{0}\left(\mathbb{Z}_{n}\right)$.

Definition 19. Let $R$ be a ring with additive identity 0 . If $a b=0$ for any $a, b \in R$, then $R$ is a zero ring.

Let $R$ be a ring with additive identity 0 . We denote by $M_{2}^{0}(R)$ the set of all $2 \times 2$ matrices of the form

$$
\left[\begin{array}{ll}
a & -a \\
a & -a
\end{array}\right], \quad a \in R
$$


It can be verified that $M_{2}^{0}(R)$ is a ring under matrix addition and matrix multiplication with additive identity

$$
\left[\begin{array}{ll}
0 & 0 \\
0 & 0
\end{array}\right]
$$

Since for any $a, b \in R$,

$$
\left[\begin{array}{ll}
a & -a \\
a & -a
\end{array}\right]\left[\begin{array}{ll}
b & -b \\
b & -b
\end{array}\right]=\left[\begin{array}{ll}
a b-a b & -a b+a b \\
a b-a b & -a b+a b
\end{array}\right]=\left[\begin{array}{ll}
0 & 0 \\
0 & 0
\end{array}\right]
$$

$M_{2}^{0}(R)$ is a zero ring. Since $\mathbb{Z}_{n}$ is a ring, it follows that $M_{2}^{0}\left(\mathbb{Z}_{n}\right)$ is a zero ring. We use $A_{i}$ to denote the matrix

$$
\left[\begin{array}{ll}
i & -i \\
i & -i
\end{array}\right], i \in \mathbb{Z}_{n}
$$

Definition 20. Let $G$ be a graph, and let $R^{0}$ be a finite zero ring. An injective function $f: V(G) \rightarrow R^{0}$ is a zero ring labeling of $G$ if $f(u)+f(v) \neq 0$ for every $u v \in E(G)$.

Given a zero ring labeling, if the order of the zero ring is equal to the order of the graph, we say that the labeling is optimal.

Example 2. Figure 2 shows a zero ring labeling of $C_{5}$ using $M_{2}^{0}\left(\mathbb{Z}_{5}\right)$. Since $\left|C_{5}\right|=$ $\left|M_{2}^{0}\left(\mathbb{Z}_{5}\right)\right|=5$, this labeling is optimal.

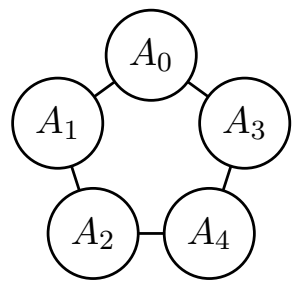

Figure 2: Zero ring labeling of $C_{5}$ using $M_{2}^{0}\left(\mathbb{Z}_{5}\right)$

Given a graph $G$ with zero ring labeling $f$, we consider the set $K=\{f(u)+f(v)$ : $u v \in E(G)\}$. Since it was shown in [1] that every graph admits a zero ring labeling, we are interested in finding zero ring labelings for $G$ such that $|K|$ is as small as possible.

Definition 21. Let $G$ be a graph with zero ring labeling $f$, and let $K=\{f(u)+f(v): u v \in$ $E(G)\}$. A zero ring labeling $f$ of $G$ is a $\boldsymbol{k}$-zero ring labeling if $|K|=k$. If $|K|=\Delta(G)$, then the zero ring labeling is efficient.

Example 3. Figure 3 shows a 4-zero ring labeling of the diamond graph using $M_{2}^{0}\left(\mathbb{Z}_{8}\right)$. In this labeling, the set of sums is $K=\left\{A_{1}, A_{2}, A_{5}, A_{7}\right\}$ and thus $|K|=4$. Figure 4 shows an efficient zero ring labeling of the butterfly graph $G$ using $M_{2}^{0}\left(\mathbb{Z}_{10}\right)$. In this labeling, the set of sums is $K=\left\{A_{2}, A_{3}, A_{7}, A_{9}\right\}$ and thus $|K|=\Delta(G)=4$. 


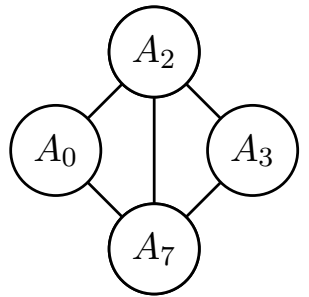

Figure 3: 4-zero ring labeling of the diamond graph using $M_{2}^{0}\left(\mathbb{Z}_{8}\right)$

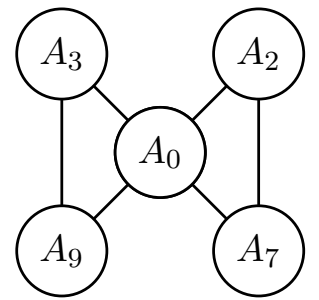

Figure 4: Efficient zero ring labeling of the butterfly graph using $M_{2}^{0}\left(\mathbb{Z}_{10}\right)$

\section{Results}

Theorem 1. Let $G$ be a graph, and let $f: G \rightarrow R^{0}$ be a zero ring labeling. If $K=$ $\{f(u)+f(v): u v \in E(G)\}$, then $\Delta(G) \leq|K| \leq\left|R^{0}\right|-1$.

Proof. Since $f$ is a zero ring labeling, $0 \notin K$. Furthermore, $R^{0}$ contains every sum $f(u)+f(v)$ where $u v \in E(G)$, hence $K \subset R^{0}$ and $|K| \leq\left|R^{0}\right|-1$.

Let $v \in V(G)$ and let $N(v)=\left\{v_{1}, v_{2}, \ldots, v_{m}\right\}$ be the set of vertices that are adjacent to $v$. Since $f$ is injective, it follows that the number of distinct sums $f(v)+f\left(v_{i}\right)$ for $i=1,2,3, \ldots, m$ generated from edges that end at $v$ is equal to $|N(v)|=m$, which is the degree of $v$. Clearly, the maximum degree of $G$ is the minimum number of elements of $K$.

Theorem 2. If $G$ is a graph with an efficient zero ring labeling, then any edge-induced subgraph $H$ of $G$ such that $\Delta(G)=\Delta(H)$ has an efficient zero ring labeling.

Proof. Let $G$ be a graph with efficient zero ring labeling $f: V(G) \rightarrow R^{0}$, and let $H$ be an edge-induced subgraph of $G$ such that $\Delta(G)=\Delta(H)$. Consider the restriction function $\left.f\right|_{H}$ of $f$ to the vertex set of $H$; that is, $\left.f\right|_{H}(v)=f(v)$ for all $v \in V(H)$. Since $f$ is injective, $\left.f\right|_{H}$ is injective. Also, $0 \notin K=\{f(u)+f(v): u v \in E(G)\}$ implies that $0 \notin K_{H}=\left\{\left.f\right|_{H}(u)+\left.f\right|_{H}(v): u v \in E(H)\right\}$. It remains to show that $\left|K_{H}\right|=\Delta(H)$.

Since $\Delta(G)=\Delta(H)$, there exists at least one vertex $v$ in $V(G)$ where $d(v)=\Delta(G)$ such that $v$ is also in $H$ where $d(v)=\Delta(G)$. Then $\left|K_{H}\right|=\Delta(G)=\Delta(H)$.

Theorem 3. Let $G$ be a graph with $n$ vertices, and let $f: V(G) \rightarrow R^{0}$ be a zero ring labeling. If $f$ is optimal and $\Delta(G)=n-1$, then $f$ is efficient.

Proof. Let $K=\{f(u)+f(v): u v \in E(G)\}$. To show that $f$ is efficient, we need to show that $|K|=n-1$.

Since $f$ is optimal, $\left|R^{0}\right|=n$. By Theorem $1, \Delta(G) \leq|K| \leq\left|R^{0}\right|-1$. But $\Delta(G)=$ $\left|R^{0}\right|-1=n-1$. Therefore, $|K|=n-1$.

We now look at the efficient zero ring labeling of some classes of trees and other common classes of graphs.

Theorem 4. Let $G$ be a caterpillar in which each vertex in the central path has an equal number of hanging leaves. Then $G$ has an efficient zero ring labeling. 
Proof. Let $\left[w_{1}, w_{2}, \ldots, w_{n}\right]$ denote the central path of a caterpillar $G$ and suppose that each $w_{i}, 1 \leq i \leq n$, has $r$ hanging leaves. Denote the hanging leaves of $w_{i}$ by $w_{i}^{j}$, where $j=1,2, \ldots, r$.

Case 1: Suppose $n=1$. In this case, $\Delta(G)=d\left(w_{1}\right)=r$. Define a function $f: V(G) \rightarrow$ $M_{2}^{0}\left(\mathbb{Z}_{r+1}\right)$ such that $f\left(w_{1}\right)=A_{0}$ and $f\left(w_{1}^{j}\right)=A_{j}$.

Clearly, $f$ is injective. Let $K=\{f(u)+f(v): u v \in E(G)\}$. To show that $f$ is an efficient zero ring labeling of $G$, we need to show that $|K|=r$ and $A_{0} \notin K$. For the set of sums, we obtain

$$
f\left(w_{1}\right)+f\left(w_{1}^{j}\right)=A_{0}+A_{j}=A_{j} \neq A_{0}
$$

for $j=1,2, \ldots, r$. Then $A_{0} \notin K$. Moreover,

$$
K=\left\{A_{1}, A_{2}, \ldots, A_{r}\right\}
$$

and thus $|K|=r$. Therefore, $f$ is an efficient zero ring labeling of $G$.

Case 2: Suppose $n=2$. In this case, $\Delta(G)=d\left(w_{1}\right)=d\left(w_{2}\right)=r+1$. Define a function $f: V(G) \rightarrow M_{2}^{0}\left(\mathbb{Z}_{2 r+2}\right)$ such that $f\left(w_{i}\right)=A_{i(r+1)}$ and $f\left(w_{i}^{j}\right)=A_{(i-1)(r+1)+j}$.

Clearly, $f$ is injective. Let $K=\{f(u)+f(v): u v \in E(G)\}$. To show that $f$ is an efficient zero ring labeling of $G$, we need to show that $|K|=r+1$ and $A_{0} \notin K$. For the set of sums, we obtain

$$
f\left(w_{i}\right)+f\left(w_{i}^{j}\right)=A_{i(r+1)}+A_{(i-1)(r+1)+j}=A_{2 i r+2 i-r-1+j}=A_{j-r-1}
$$

for $j=1,2, \ldots, r$, and

$$
f\left(w_{1}\right)+f\left(w_{2}\right)=A_{r+1}+A_{2 r+2}=A_{3 r+3}=A_{r+1} \neq A_{0}
$$

It remains to show that $A_{j-r-1} \neq A_{0}$ for $j=1,2, \ldots, r$. By substitution, we obtain the sums $A_{-r}, A_{1-r}, \ldots, A_{-1}$, which are equal to $A_{r+2}, A_{r+3}, \ldots, A_{2 r+1}$, respectively. For $r+2 \leq m \leq 2 r+1$, we have $0<m<2 r+2$, hence $m \neq \equiv 0(\bmod 2 r+2)$. Thus, $A_{m} \neq A_{0}$ for $m=r+2, r+3, \ldots, 2 r+1$. Then $A_{0} \notin K$. Moreover,

$$
K=\left\{A_{r+1}, A_{r+2}, A_{r+3}, \ldots, A_{2 r+1}\right\}
$$

and thus $|K|=r+1$. Therefore, $f$ is an efficient zero ring labeling of $G$.

Case 3: Suppose $n \geq 3$. In this case, $\Delta(G)=d\left(w_{i}\right)$, where $i \neq 1$ and $i \neq n$. Then $\Delta(G)=r+2$. Define a function $f: V(G) \rightarrow M_{2}^{0}\left(\mathbb{Z}_{n r+n}\right)$ such that

$$
f\left(w_{i}\right)= \begin{cases}A_{(r+1)\left(\frac{2 n-i-1}{2}\right)} & \text { if } i \text { is odd } \\ A_{(r+1)\left(\frac{i-2}{2}\right)} & \text { if } i \text { is even }\end{cases}
$$

and

$$
f\left(w_{i}^{j}\right)=\left\{\begin{array}{ll}
A_{(r+1)\left(\frac{i-3}{2}\right)+j} & \text { if } i \text { is odd } \\
A_{(r+1)\left(\frac{2 n-i-2}{2}\right)+j} & \text { if } i \text { is even }
\end{array} .\right.
$$


Clearly, $f$ is injective. Let $K=\{f(u)+f(v): u v \in E(G)\}$. To show that $f$ is an efficient zero ring labeling of $G$, we need to show that $|K|=r+2$ and $A_{0} \notin K$. For adjacent vertices in the central path, we obtain the sums

$$
f\left(w_{i}\right)+f\left(w_{i+1}\right)=A_{(r+1)\left(\frac{2 n-i-1}{2}\right)}+A_{(r+1)\left(\frac{(i+1)-2}{2}\right)}=A_{(r+1)(n-1)}
$$

if $i$ is odd, and

$$
f\left(w_{i}\right)+f\left(w_{i+1}\right)=A_{(r+1)\left(\frac{i-2}{2}\right)}+A_{(r+1)\left(\frac{2 n-(i+1)-1}{2}\right)}=A_{(r+1)(n-2)}
$$

if $i$ is even. Note that $A_{(r+1)(n-1)} \neq A_{0}$; otherwise, either $r \equiv-1(\bmod n r+n)$, which is a contradiction by definition of $r$, or $n \equiv 1(\bmod n r+n)$, which is also a contradiction by definition of $n$ in this case. Similarly, $A_{(r+1)(n-2)} \neq A_{0}$.

For pairs of adjacent hanging leaf and vertex in the central path, we obtain the sums

$$
f\left(w_{i}\right)+f\left(w_{i}^{j}\right)=A_{(r+1)\left(\frac{2 n-i-1}{2}\right)}+A_{(r+1)\left(\frac{i-3}{2}\right)+j}=A_{(r+1)(n-2)+j}
$$

for $j=1,2, \ldots, r$ if $i$ is odd, and

$$
f\left(w_{i}\right)+f\left(w_{i}^{j}\right)=A_{(r+1)\left(\frac{i-2}{2}\right)}+A_{(r+1)\left(\frac{2 n-i-2}{2}\right)+j}=A_{(r+1)(n-2)+j}
$$

for $j=1,2, \ldots, r$ if $i$ is even. We show that $A_{(r+1)(n-2)+j} \neq A_{0}$. Assume that $A_{(r+1)(n-2)+j}=$ $A_{0}$ for some $j$. But this implies that $j \equiv 2 r+2(\bmod n r+n)$. This is a contradiction since $n$ is at least 3 in this case, and $j$ cannot be greater than $r$ by its definition. Then $A_{0} \notin K$. Moreover,

$$
K=\left\{A_{(r+1)(n-2)}, A_{(r+1)(n-2)+1}, \ldots, A_{(r+1)(n-2)+r}, A_{(r+1)(n-1)}\right\}
$$

and thus $|K|=r+2$. Therefore, $f$ is an efficient zero ring labeling of $G$.

Example 4. Figure 5 shows an efficient zero ring labeling of a caterpillar $G$ with a central path with four vertices, where each vertex in the central path has three hanging leaves, using $M_{2}^{0}\left(\mathbb{Z}_{16}\right)$. In this labeling, the set of sums is $K=\left\{A_{8}, A_{9}, A_{10}, A_{11}, A_{12}\right\}$ and thus $|K|=\Delta(G)=5$.

Corollary 1. A path graph has an efficient zero ring labeling.

Proof. Let $G$ be the path graph $P_{n}$. Consider $G$ as its own central path. Then $G$ is a caterpillar with a central path with $n$ vertices, where each vertex in the central path has no hanging leaf. By Theorem $4, G$ has an efficient zero ring labeling.

Example 5. Figure 6 shows an efficient zero ring labeling of $P_{10}$ using $M_{2}^{0}\left(\mathbb{Z}_{10}\right)$. In this labeling, the set of sums is $K=\left\{A_{8}, A_{9}\right\}$ and thus $|K|=\Delta\left(P_{10}\right)=2$. 


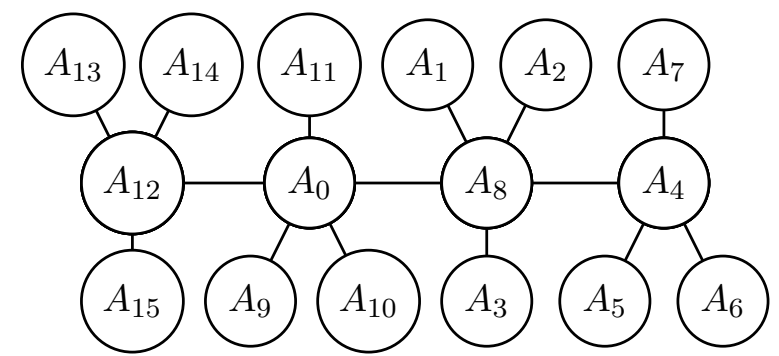

Figure 5: Efficient zero ring labeling of a caterpillar using $M_{2}^{0}\left(\mathbb{Z}_{16}\right)$

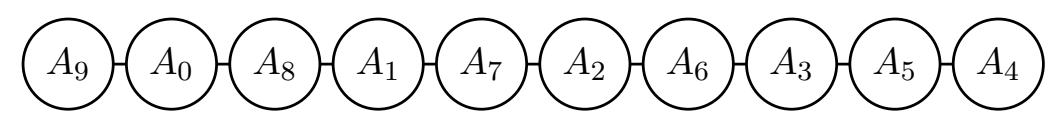

Figure 6: Efficient zero ring labeling of $P_{10}$ using $M_{2}^{0}\left(\mathbb{Z}_{10}\right)$

Corollary 2. A star graph has an efficient zero ring labeling.

Proof. Let $G$ be the star graph $S_{n}$. Consider the center of $G$ as its central path. Then $G$ is a caterpillar with a central path with one vertex, and this vertex has $n-1$ hanging leaves. Vacuously, each vertex in the central path of $G$ has an equal number of hanging leaves. By Theorem 4, $G$ has an efficient zero ring labeling.

Example 6. Figure 7 shows an efficient zero ring labeling of $S_{9}$ using $M_{2}^{0}\left(\mathbb{Z}_{9}\right)$. In this labeling, the set of sums is $K=\left\{A_{1}, A_{2}, A_{3}, A_{4}, A_{5}, A_{6}, A_{7}, A_{8}\right\}$ and thus $|K|=\Delta\left(S_{9}\right)=8$.

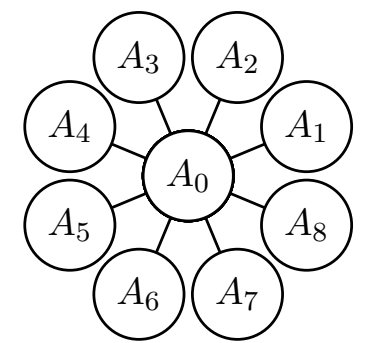

Figure 7: Efficient zero ring labeling of $S_{9}$ using $M_{2}^{0}\left(\mathbb{Z}_{9}\right)$

Corollary 3. A bistar has an efficient zero ring labeling.

Proof. Let $G$ be the bistar $B_{n}$. Consider the path joining the centers of the two star graphs $S_{n}$ in $G$ as its central path. Then $G$ is a caterpillar with a central path with two vertices, where each vertex in the central path has $n-1$ hanging leaves. By Theorem 4 , $G$ has an efficient zero ring labeling.

Example 7. Figure 8 shows an efficient zero ring labeling of $B_{9}$ using $M_{2}^{0}\left(\mathbb{Z}_{18}\right)$. In this labeling, the set of sums is $K=\left\{A_{9}, A_{10}, A_{11}, A_{12}, A_{13}, A_{14}, A_{15}, A_{16}, A_{17}\right\}$ and thus $|K|=\Delta\left(B_{9}\right)=9$. 


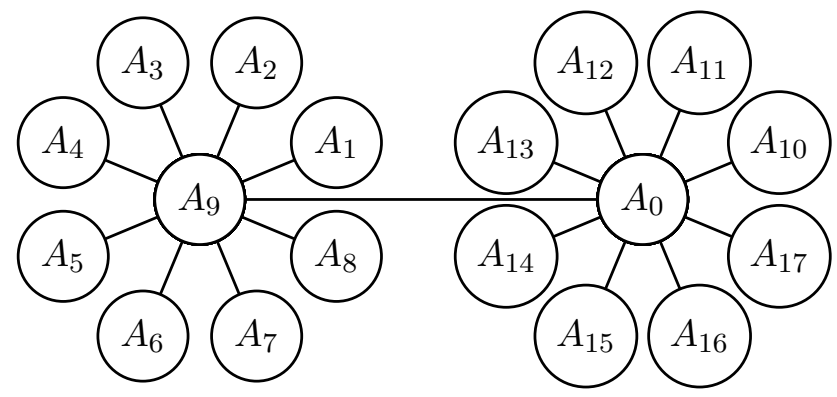

Figure 8: Efficient zero ring labeling of $B_{9}$ using $M_{2}^{0}\left(\mathbb{Z}_{18}\right)$

Theorem 5. A centipede graph has an efficient zero ring labeling.

Proof. Let $G$ be an $n$-centipede. By definition of a centipede, we can find a central path in $G$ such that each vertex in the central path has 1 hanging leaf. By Theorem $4, G$ has an efficient zero ring labeling.

Example 8. Figure 9 shows an efficient zero ring labeling of a 6 -centipede using $M_{2}^{0}\left(\mathbb{Z}_{12}\right)$. In this labeling, the set of sums is $K=\left\{A_{8}, A_{9}, A_{10}\right\}$ and thus $|K|=3$, which is the maximum degree of the graph.

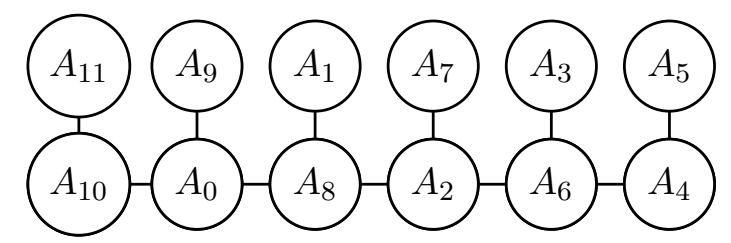

Figure 9: Efficient zero ring labeling of a 6-centipede using $M_{2}^{0}\left(\mathbb{Z}_{12}\right)$

Lemma 1. Let $G$ be a caterpillar with at least 3 vertices. Then $G$ is a caterpillar with respect to some central path $\left[w_{1}, w_{2}, \ldots, w_{n}\right]$, where $n \geq 3$ and $w_{1}$ and $w_{n}$ have no hanging leaf.

Proof. Let $G$ be a caterpillar with at least 3 vertices, and let $\left[v_{1}, v_{2}, \ldots, v_{m}\right]$ denote its central path. Suppose each vertex $v_{i}$ has $r_{i}$ hanging leaves, and let $v_{i}^{j}$, where $j=$ $1,2, \ldots, r_{i}$, denote the hanging leaves of $v_{i}$.

Consider $v_{1}, v_{2}, \ldots, v_{m}$ as vertices in a central path $P$, along with $v_{1}^{1}$ if $r_{1} \geq 1$, and $v_{m}^{r_{m}}$ if $r_{m} \geq 1$. Thus, $P$ is one of the following: $\left[v_{1}, v_{2}, \ldots, v_{m}\right],\left[v_{1}^{1}, v_{1}, v_{2}, \ldots, v_{m}\right]$, $\left[v_{1}, v_{2}, \ldots, v_{m}, v_{m}^{r_{m}}\right],\left[v_{1}^{1}, v_{1}, v_{2}, \ldots, v_{m}, v_{m}^{r_{m}}\right]$. In any case, the number of vertices in $P$ is at least three, and its endvertices have no hanging leaf. Clearly, each vertex in $G$ is within distance one from $P$ and thus $G$ is a caterpillar with respect to central path $P$.

Theorem 6. A caterpillar has an efficient zero ring labeling. 
Proof. Let $G$ be a caterpillar with at least three vertices. By Lemma 1, $G$ is a caterpillar with respect to some central path $\left[w_{1}, w_{2}, \ldots, w_{n}\right]$, where $n \geq 3$ and $w_{1}$ and $w_{n}$ have no hanging leaf. For $i=2,3, \ldots, n-1$, suppose $w_{i}$ has $r_{i}$ hanging leaves, and let $w_{i}^{j}$, where $j=1,2, \ldots, r_{i}$, denote the hanging leaves of $w_{i}$.

Suppose $r_{k}$ is the maximum number of hanging leaves of a vertex in the central path; that is, $r_{k} \geq r_{i}$ for $i=2,3, \ldots, n-1$. Then $\Delta(G)=r_{k}+2$.

Consider a caterpillar $H$ with a central path with $n$ vertices, and where each vertex in the central path has $r_{k}$ hanging leaves. Let $\left[v_{1}, v_{2}, \ldots, v_{n}\right]$ denote its central path, and let $v_{i}^{j}$, where $j=1,2, \ldots, r_{k}$, denote the hanging leaves of $v_{i}$. By Theorem $4, H$ has an efficient zero ring labeling $h$.

Define a function $f: V(G) \rightarrow M_{2}^{0}\left(\mathbb{Z}_{n r_{k}+n-r_{k}}\right)$ such that $f\left(w_{i}\right)=h\left(v_{i}\right)$ and $f\left(w_{i}^{j}\right)=$ $h\left(w_{i}^{j}\right)$. Since $h$ is injective, it follows that $f$ is also injective. $G$ is an edge-induced subgraph of $H$, so $A_{0} \notin K_{H}=\{h(u)+h(v): u v \in E(H)\}$ implies that $A_{0} \notin K=$ $\{f(u)+f(v): u v \in E(G)\}$. To show that $f$ is an efficient zero ring labeling of $G$, it remains to show that $|K|=\Delta(G)$. Using the labeling in the proof of Theorem 4, we obtain

$$
K=\left\{A_{\left(r_{k}+1\right)(n-2)}, A_{\left(r_{k}+1\right)(n-2)+1}, \ldots, A_{\left(r_{k}+1\right)(n-2)+r_{k}}, A_{\left(r_{k}+1\right)(n-1)}\right\} .
$$

Thus, $|K|=r_{k}+2=\Delta(G)$.

Example 9. Figure 10 shows an efficient zero ring labeling of a caterpillar $G$ with maximum degree $\Delta(G)=6$ using $M_{2}^{0}\left(\mathbb{Z}_{31}\right)$. In this labeling, the set of sums is $K=$ $\left\{A_{15}, A_{16}, A_{17}, A_{18}, A_{19}, A_{20}\right\}$ and thus $|K|=\Delta(G)=6$.

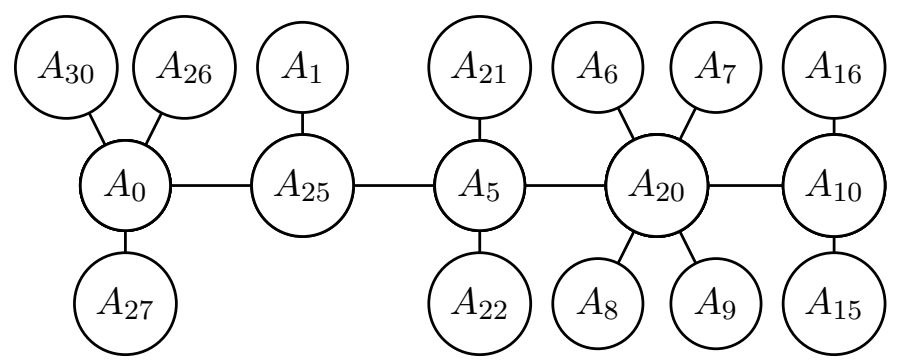

Figure 10: Efficient zero ring labeling of a caterpillar using $M_{2}^{0}\left(\mathbb{Z}_{31}\right)$

Theorem 7. A spider has an efficient zero ring labeling.

Proof. Let $G$ be a spider with $n$ legs, where $n \geq 3$. Then $\Delta(G)=n$. Let the head of $G$ be denoted by $w$, and let $w_{1}^{1}, w_{2}^{1}, \ldots, w_{n}^{1}$ denote the vertices that are adjacent to $w$. Suppose each leg containing $w_{i}^{1}$ has $r_{i}$ vertices, and let $\left[w_{i}^{1}, w_{i}^{2}, \ldots, w_{i}^{r_{i}}\right]$ denote the leg containing $w_{i}^{1}$. 
Suppose $r_{k}=\max \left\{r_{1}, r_{2}, \ldots, r_{n}\right\}$. Define a function $f: V(G) \rightarrow M_{2}^{0}\left(\mathbb{Z}_{(n-1)\left(2 r_{k}+1\right)}\right)$ such that $f(w)=A_{0}$,

$$
f\left(w_{i}^{j}\right)= \begin{cases}A_{(n-1)}\left(\frac{2 r_{k}-j+1}{2}\right)+i-1 & \text { if } j \text { is odd } \\ A_{(n-1)\left(\frac{j}{2}\right)-i+1} & \text { if } j \text { is even }\end{cases}
$$

for $i=1,2, \ldots, n-1$, and

$$
f\left(w_{n}^{j}\right)=\left\{\begin{array}{ll}
A_{(n-1)}\left(\frac{2 r_{k}+j+1}{2}\right) & \text { if } j \text { is odd } \\
A_{(n-1)\left(\frac{4 r_{k}-j+2}{2}\right)} & \text { if } j \text { is even }
\end{array} .\right.
$$

Clearly, $f$ is injective. Let $K=\{f(u)+f(v): u v \in E(G)\}$. To show that $f$ is an efficient zero ring labeling of $G$, we need to show that $|K|=n$ and $A_{0} \notin K$. For pairs of adjacent head and vertex in a leg, we obtain the sums

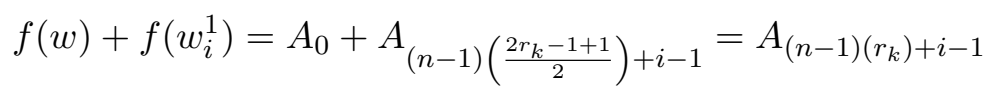

for $i=1,2, \ldots, n-1$, and

$$
f(w)+f\left(w_{n}^{1}\right)=A_{0}+A_{(n-1)\left(r_{k}+1\right)}=A_{(n-1)\left(r_{k}+1\right)}=A_{(n-1)\left(r_{k}\right)+n-1} .
$$

Thus, we have the sums

$$
A_{(n-1)\left(r_{k}\right)}, A_{(n-1)\left(r_{k}\right)+1}, A_{(n-1)\left(r_{k}\right)+2}, \ldots, A_{(n-1)\left(r_{k}\right)+n-2}, A_{(n-1)\left(r_{k}\right)+n-1} .
$$

For adjacent vertices in a leg, we obtain the sums

$$
\begin{aligned}
f\left(w_{i}^{j}\right)+f\left(w_{i}^{j+1}\right) & =A_{(n-1)\left(\frac{2 r_{k}-j+1}{2}\right)+i-1}+A_{(n-1)\left(\frac{j+1}{2}\right)-i+1} \\
& =A_{(n-1)\left(r_{k}+1\right)}=A_{(n-1)\left(r_{k}\right)+n-1}
\end{aligned}
$$

if $j$ is odd, and

$$
f\left(w_{i}^{j}\right)+f\left(w_{i}^{j+1}\right)=A_{(n-1)\left(\frac{j}{2}\right)-i+1}+A_{(n-1)\left(\frac{2 r_{k}-(j+1)+1}{2}\right)+i-1}=A_{(n-1)\left(r_{k}\right)}
$$

if $j$ is even, for $i=1,2, \ldots, n-1$. Moreover, we have

$$
\begin{aligned}
f\left(w_{n}^{j}\right)+f\left(w_{n}^{j+1}\right) & =A_{(n-1)\left(\frac{2 r_{k}+j+1}{2}\right)}+A_{(n-1)\left(\frac{4 r_{k}-(j+1)+2}{2}\right)}=A_{(n-1)\left(3 r_{k}+1\right)} \\
& =A_{(n-1)\left(r_{k}\right)}
\end{aligned}
$$

if $j$ is odd, and

$$
\begin{aligned}
f\left(w_{n}^{j}\right)+f\left(w_{n}^{j+1}\right) & =A_{(n-1)\left(\frac{4 r_{k}-j+2}{2}\right)}+A_{(n-1)\left(\frac{2 r_{k}+(j+1)+1}{2}\right)}=A_{(n-1)\left(3 r_{k}+2\right)} \\
& =A_{(n-1)\left(r_{k}+1\right)}=A_{(n-1)\left(r_{k}\right)+n-1}
\end{aligned}
$$


if $j$ is even. Then

$$
K=\left\{A_{(n-1)\left(r_{k}\right)}, A_{(n-1)\left(r_{k}\right)+1}, \ldots, A_{(n-1)\left(r_{k}\right)+n-2}, A_{(n-1)\left(r_{k}\right)+n-1}\right\},
$$

hence $|K|=n$. For $m=0,1,2, \ldots, n-1$, it is clear that $(n-1)\left(r_{k}\right)+m>0$ and $(n-1)\left(r_{k}\right)+m<(n-1)\left(r_{k}\right)+(n-1)\left(r_{k}+1\right)=(n-1)\left(2 r_{k}+1\right)$. Then $A_{0} \notin K$. Therefore, $f$ is an efficient zero ring labeling of $G$.

Example 10. Figure 11 shows an efficient zero ring labeling of a spider with 4 legs using $M_{2}^{0}\left(\mathbb{Z}_{33}\right)$. In this labeling, the set of sums is $K=\left\{A_{15}, A_{16}, A_{17}, A_{18}\right\}$ and thus $|K|=4$.

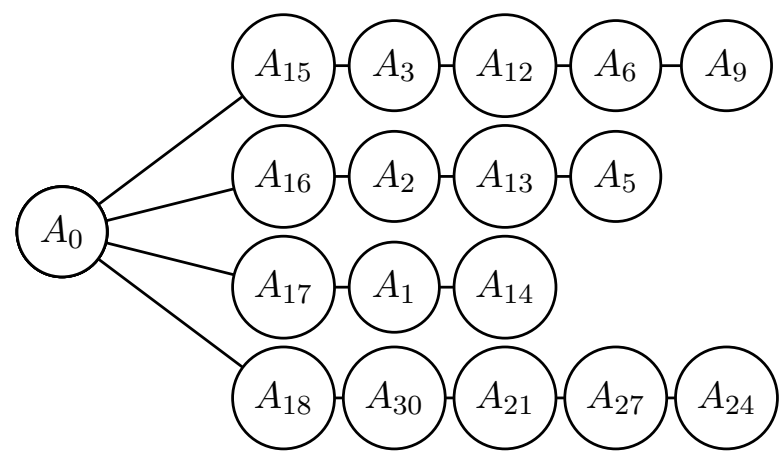

Figure 11: Efficient zero ring labeling of a spider using $M_{2}^{0}\left(\mathbb{Z}_{33}\right)$

Lemma 2. Let $G$ be a lobster with at least three vertices. Then $G$ is a lobster with respect to some central path $\left[v_{1}, v_{2}, \ldots, v_{m}\right]$, where $m \geq 3$ and $v_{1}$ and $v_{m}$ have no elbow.

Proof. Let $G$ be a lobster with at least three vertices, and let $\left[w_{1}, w_{2}, \ldots, w_{n}\right]$ denote its central path. Suppose each vertex $w_{i}$ has $r_{i}$ elbows, and let $w_{i, j}$, where $j=1,2, \ldots, r_{i}$, denote the elbows of $w_{i}$. Suppose each elbow $w_{i, j}$ has $s_{i, j}$ hanging leaves, and let $w_{i, j, k}$, where $k=1,2, \ldots, s_{i, j}$, denote the hanging leaves of $w_{i, j}$.

Consider $w_{1}, w_{2}, \ldots, w_{n}$ as vertices in a central path $P$, along with $w_{1,1}$ if $r_{1} \geq 1, w_{1,1,1}$ if $s_{1,1} \geq 1, w_{n, 1}$ if $r_{n} \geq 1$, and $w_{n, 1,1}$ if $s_{n, 1} \geq 1$. Thus, $P$ is one of the following:

- $\left[w_{1}, w_{2}, \ldots, w_{n}\right]$

- $\left[w_{1,1}, w_{1}, w_{2}, \ldots, w_{n}\right]$

- $\left[w_{1,1,1}, w_{1,1}, w_{1}, w_{2}, \ldots, w_{n}\right]$

- $\left[w_{1}, w_{2}, \ldots, w_{n}, w_{n, 1}\right]$

- $\left[w_{1,1}, w_{1}, w_{2}, \ldots, w_{n}, w_{n, 1}\right]$

- $\left[w_{1,1,1}, w_{1,1}, w_{1}, w_{2}, \ldots, w_{n}, w_{n, 1}\right]$

- $\left[w_{1}, w_{2}, \ldots, w_{n}, w_{n, 1}, w_{n, 1,1}\right]$ 
- $\left[w_{1,1}, w_{1}, w_{2}, \ldots, w_{n}, w_{n, 1}, w_{n, 1,1}\right]$

- $\left[w_{1,1,1}, w_{1,1}, w_{1}, w_{2}, \ldots, w_{n}, w_{n, 1}, w_{n, 1,1}\right]$

In any case, the number of vertices in $P$ is at least three, and its endvertices have no elbow. Clearly, each vertex in $G$ is within distance two from $P$ and thus $G$ is a lobster with respect to central path $P$.

Theorem 8. Let $G$ be a lobster in which each elbow has at most one hanging leaf. Then $G$ has an efficient zero ring labeling.

Proof. Let $G$ be a lobster with at least three vertices in which each elbow has at most one hanging leaf. By Lemma 2, $G$ is a lobster with respect to some central path $\left[w_{1}, w_{2}, \ldots, w_{n}\right]$, where $n \geq 3$ and $w_{1}$ and $w_{n}$ have no elbow. Clearly, each elbow has at most one hanging leaf with respect to this central path.

Suppose each vertex $w_{i}$ has $r_{i}$ elbows, and let $w_{i, j}$, where $j=1,2, \ldots, r_{i}$, denote the elbows of $w_{i}$. If it exists, let $w_{i, j, 1}$ denote the hanging leaf of $w_{i, j}$.

Suppose $r_{k}$ is the maximum number of elbows of a vertex in the central path; that is, $r_{k} \geq r_{i}$ for $i=1,2, \ldots, n$. Then $\Delta(G)=r_{k}+2$. Define a function $f: V(G) \rightarrow$ $M_{2}^{0}\left(\mathbb{Z}_{2 n r_{k}+n-2 r_{k}}\right)$ such that

$$
\begin{gathered}
f\left(w_{i}\right)= \begin{cases}A_{\left(2 r_{k}+1\right)\left(\frac{2 n-i-1}{2}\right)} & \text { if } i \text { is odd } \\
A_{\left(2 r_{k}+1\right)\left(\frac{i-2}{2}\right)} & \text { if } i \text { is even }\end{cases} \\
f\left(w_{i, j}\right)= \begin{cases}A_{\left(2 r_{k}+1\right)\left(\frac{i-3}{2}\right)+j} & \text { if } i \text { is odd } \\
A_{\left(2 r_{k}+1\right)\left(\frac{2 n-i-2}{2}\right)+j} & \text { if } i \text { is even. }\end{cases}
\end{gathered}
$$

and

$$
f\left(w_{i, j, 1}\right)=\left\{\begin{array}{ll}
A_{\left(2 r_{k}+1\right)\left(\frac{2 n-i+1}{2}\right)-j} & \text { if } i \text { is odd } \\
A_{\left(2 r_{k}+1\right)\left(\frac{i}{2}\right)-j} & \text { if } i \text { is even }
\end{array} .\right.
$$

Clearly, $f$ is injective. Let $K=\{f(u)+f(v): u v \in E(G)\}$. To show that $f$ is an efficient zero ring labeling of $G$, we need to show that $|K|=r_{k}+2$ and $A_{0} \notin K$. For adjacent vertices in the central path, we obtain the sums

$$
f\left(w_{i}\right)+f\left(w_{i+1}\right)=A_{\left(2 r_{k}+1\right)\left(\frac{2 n-i-1}{2}\right)}+A_{\left(2 r_{k}+1\right)\left(\frac{(i+1)-2}{2}\right)}=A_{\left(2 r_{k}+1\right)(n-1)}
$$

if $i$ is odd, and

$$
f\left(w_{i}\right)+f\left(w_{i+1}\right)=A_{\left(2 r_{k}+1\right)\left(\frac{2 n-i-1}{2}\right)}+A_{\left(2 r_{k}+1\right)\left(\frac{2 n-(i+1)-1}{2}\right)}=A_{\left(2 r_{k}+1\right)(n-2)}
$$

if $i$ is even. Thus, there are only two distinct sums obtained for adjacent vertices in the central path. For pairs of adjacent elbow and vertex in the central path, we obtain the sums

$$
f\left(w_{i}\right)+f\left(w_{i, j}\right)=A_{\left(2 r_{k}+1\right)\left(\frac{i-2}{2}\right)}+A_{\left(2 r_{k}+1\right)\left(\frac{i-3}{2}\right)+j}=A_{\left(2 r_{k}+1\right)(n-2)+j}
$$


for $j=1,2, \ldots, r_{i}$ if $i$ is odd, and

$$
f\left(w_{i}\right)+f\left(w_{i, j}\right)=A_{\left(2 r_{k}+1\right)\left(\frac{i-2}{2}\right)}+A_{\left(2 r_{k}+1\right)\left(\frac{i-3}{2}\right)+j}=A_{\left(2 r_{k}+1\right)(n-2)+j}
$$

for $j=1,2, \ldots, r_{i}$ if $i$ is even. Since $r_{k}$ is the maximum number of elbows of a vertex in the central path, the obtained sums for pairs of adjacent elbow and vertex in the central path are $A_{\left(2 r_{k}+1\right)(n-2)+1}, A_{\left(2 r_{k}+1\right)(n-2)+2}, \ldots, A_{\left(2 r_{k}+1\right)(n-2)+r_{k}}$. For pairs of adjacent hanging leaf and elbow, we obtain the sums

$$
f\left(w_{i, j}\right)+f\left(w_{i, j, 1}\right)=A_{\left(2 r_{k}+1\right)\left(\frac{i-3}{2}\right)+j}+A_{\left(2 r_{k}+1\right)\left(\frac{2 n-i+1}{2}\right)-j}=A_{\left(2 r_{k}+1\right)(n-1)}
$$

if $i$ is odd, and

$$
f\left(w_{i, j}\right)+f\left(w_{i, j, 1}\right)=A_{\left(2 r_{k}+1\right)\left(\frac{2 n-i-2}{2}\right)+j}+A_{\left(2 r_{k}+1\right)\left(\frac{i}{2}\right)-j}=A_{\left(2 r_{k}+1\right)(n-1)}
$$

if $i$ is even. Thus, the sum of any pair of adjacent elbow and hanging leaf is equal. Then

$$
K=\left\{A_{\left(2 r_{k}+1\right)(n-2)}, A_{\left(2 r_{k}+1\right)(n-2)+1}, \ldots, A_{\left(2 r_{k}+1\right)(n-2)+r_{k}}, A_{\left(2 r_{k}+1\right)(n-1)}\right\},
$$

hence $|K|=r_{k}+2$.

To show that $A_{0} \notin K$, it is sufficient to show that $\left(2 r_{k}+1\right)(n-2),\left(2 r_{k}+1\right)(n-2)+$ $1,\left(2 r_{k}+1\right)(n-2)+2, \ldots,\left(2 r_{k}+1\right)(n-2)+r_{k},\left(2 r_{k}+1\right)(n-1)$ are greater than 0 but less than $2 n r_{k}+n-2 r_{k}$. The central path has at least 3 vertices, so $n \geq 3$, and $r_{k}$ is at least zero by its definition. Then $\left(2 r_{k}+1\right)(n-2)+m$ for $m=0,1, \ldots, r_{k}$ and $\left(2 r_{k}+1\right)(n-1)$ are greater than zero. Moreover, $\left(2 r_{k}+1\right)(n-2)+m<\left(2 r_{k}+1\right)(n-2)+\left(2 r_{k}+1\right)=\left(2 r_{k}+1\right)(n-1)$ for $m=0,1, \ldots, r_{k}$. Since $\left(2 r_{k}+1\right)(n-1)=\left(2 r_{k}+1\right)(n)-2 r_{k}-1<\left(2 r_{k}+1\right)(n)-2 r_{k}=$ $2 n r_{k}+n-2 r_{k}$, it follows that $A_{0} \notin K$.

Example 11. Figure 12 shows an efficient zero ring labeling of a lobster $G$ in which each elbow has at most one hanging leaf. In this labeling, the set of sums is $K=$ $\left\{A_{54}, A_{55}, A_{56}, A_{57}, A_{58}, A_{63}\right\}$ and thus $|K|=6=\Delta(G)$.

Theorem 9. Let $G$ be a rooted tree whose root has the maximum degree. If the height of $G$ is at most two, then $G$ has an efficient zero ring labeling.

Proof. Let $G$ be a rooted tree of height two and with root $w$ such that $d(w)=n=$ $\Delta(G)=n$. If $n$ is one or two, then $G$ is a caterpillar. Thus, by Theorem $6, G$ has an efficient zero ring labeling.

Suppose $n$ is at least three, and there exists at least one vertex of level two. Denote the children of $w$ by $w_{1}, w_{2}, \ldots, w_{n}$. Since $w$ has $n$ children and $\Delta(G)=n$, every child of $w$ has at most $n-1$ children. Suppose $w_{i}$ has $r_{i}$ children, and let $w_{i, j}$, where $j=1,2, \ldots, r_{i}$ denote the children of $w_{i}$.

Define a function $f: V(G) \rightarrow M_{2}^{0}\left(\mathbb{Z}_{2^{n+1}-1}\right)$ such that $f(w)=A_{0}, f\left(w_{i}\right)=A_{2^{i+1}-3}$, and

$$
f\left(w_{i, j}\right)= \begin{cases}A_{2^{i+j+1}-2^{i+1}} & \text { if } i+j<n+1 \\ A_{2^{n+1}-2^{i+1}+3} & \text { if } i+j=n+1 . \\ A_{2^{n+1}-2^{i+1}+2^{i+j-n+1}-1} & \text { if } i+j>n+1\end{cases}
$$




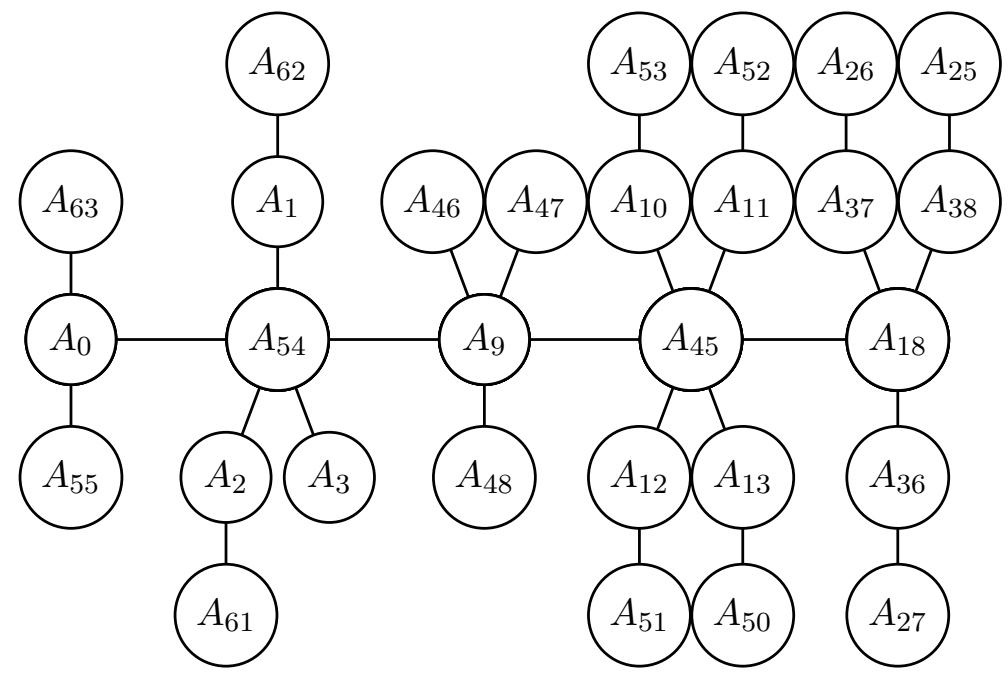

Figure 12: Efficient zero ring labeling of a lobster using $M_{2}^{0}\left(\mathbb{Z}_{64}\right)$

Clearly, $f$ is injective. Let $K=\{f(u)+f(v): u v \in E(G)\}$. To show that $f$ is an efficient zero ring labeling of $G$, we need to show that $|K|=\Delta(G)=n$ and $A_{0} \notin K$.

For pairs of adjacent root and vertex of level one, we obtain

$$
f(w)+f\left(w_{i}\right)=A_{0}+A_{2^{i+1}-3}=A_{2^{i+1}-3}
$$

for $i=1,2, \ldots, n$. Then we have the sums $A_{1}, A_{5}, \ldots, A_{2^{n+1}-3}$. For pairs of adjacent vertex of level one and vertex of level two, we have three cases.

Case 1: Suppose $i+j<n+1$. Then we obtain

$$
f\left(w_{i}\right)+f\left(w_{i}^{j}\right)=A_{2^{i+1}-3}+A_{2^{i+j+1}-2^{i+1}}=A_{2^{i+j+1}-3} .
$$

Since each of $i$ and $j$ are at least one, we have the sums $A_{2^{m+1}-3}$ for $m=2,3, \ldots, n$. These are $A_{5}, A_{13}, \ldots, A_{2^{n+1}-3}$. Note that these sums are same with those from pairs of adjacent root and vertex of level one, only that $A_{1}$ is not included.

Case 2: Suppose $i+j=n+1$. Then we obtain

$$
f\left(w_{i}\right)+f\left(w_{i}^{j}\right)=A_{2^{i+1}-3}+A_{2^{n+1}-2^{i+1}+3}=A_{2^{n+1}}=A_{1} .
$$

Thus, in this case, there is only one possible sum, which is $A_{1}$.

Case 3: Suppose $i+j>n+1$. Then we obtain

$$
\begin{aligned}
f\left(w_{i}\right)+f\left(w_{i}^{j}\right) & =A_{2^{i+1}-3}+A_{2^{n+1}-2^{i+1}+2^{i+j-n+1}-1}=A_{2^{n+1}+2^{i+j-n+1}-4} \\
& =A_{2^{i+j-n+1}-3} .
\end{aligned}
$$

Since $i+j>n+1$, it follows that $i+j$ is at least $\mathrm{n}+2$. Also, $i$ is at most $n$ and $j$ is at most $n-1$, so $i+j$ is at most $2 n-1$. Hence, $2^{i+j-n+1}-3 \geq 2^{(n+2)-n+1}-3=2^{3}-3=5$ and $2^{i+j-n+1}-3 \leq 2^{(2 n-1)-n+1}-3=2^{n}-3$. Then we have the sums $A_{5}, A_{13}, \ldots, A_{2^{n}-3}$. 
It can be observed that these sums are same with those from pairs of adjacent root and vertex of level one, only that $A_{1}$ and $A_{2^{n+1}-3}$ are not included.

Therefore,

$$
K=\left\{A_{1}, A_{5}, \ldots, A_{2^{n+1}-3}\right\},
$$

hence $|K|=n$. For $m=1,5, \ldots, 2^{n+1}-3$, it is also clear that $m>0$ and $m<2^{n+1}-1$. Thus, $A_{0} \notin K$.

Example 12. Figure 13 shows an efficient zero ring labeling of a rooted tree $G$ with a height of two. In this labeling, the set of sums is $K=\left\{A_{1}, A_{5}, A_{13}, A_{29}\right\}$ and thus $|K|=4=\Delta(G)$.

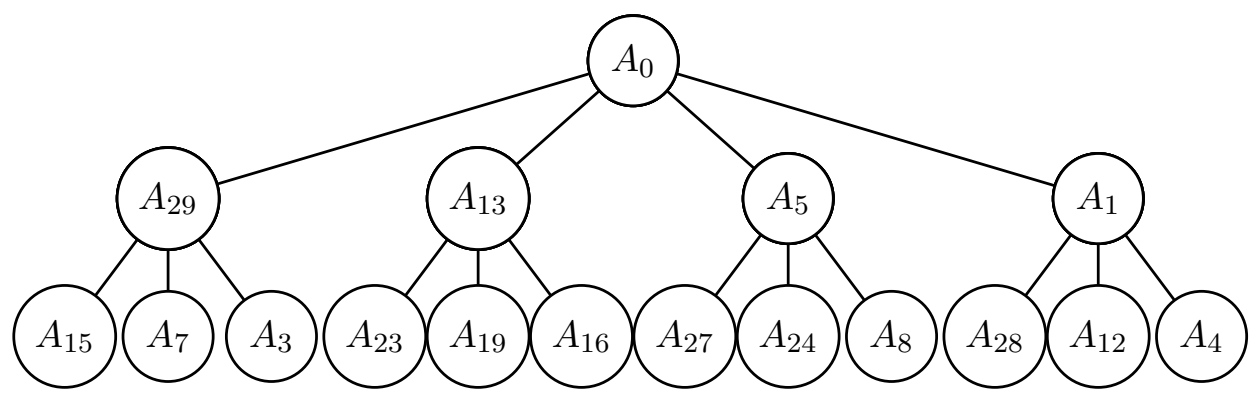

Figure 13: Efficient zero ring labeling of a rooted tree with a height of two using $M_{2}^{0}\left(\mathbb{Z}_{31}\right)$

Theorem 10. Let $G$ be a disjoint union of a finite number of caterpillars. Then $G$ has an efficient zero ring labeling.

Proof. Let $G$ be a disjoint union of $p$ caterpillars. We show that $G$ has an efficient zero ring labeling.

Case 1: The order of each caterpillar is one or two; that is, each caterpillar is either the trivial graph or $P_{2}$.

If all $p$ caterpillars are trivial, then $\Delta(G)=0$. We label the vertices by $A_{0}, A_{1}, A_{2}, \ldots, A_{p-1}$. Clearly, the labeling is injective. Moreover, the set of sums is empty and thus $A_{0} \notin K$. We are done.

Suppose at least one caterpillar is not trivial. Then $\Delta(G)=1$. Let $G_{1}, G_{2}, \ldots, G_{m}$ denote the caterpillars with two vertices, and let $G_{m+1}, G_{m+2}, \ldots, G_{p}$ denote the trivial caterpillars, where $1 \leq m \leq p$. Let $V\left(G_{i}\right)=\left\{w_{i-1}, w_{2 m-i+1}\right\}$ for $i=1,2, \ldots, m$, and let $V\left(G_{i}\right)=\left\{w_{i+m}\right\}$ for $i=m+1, m+2, \ldots, p$.

Define a function $f: V(G) \rightarrow M_{2}^{0}\left(\mathbb{Z}_{m+p+1}\right)$ such that $f\left(w_{i}\right)=A_{i}$. Let $K=$ $\{f(u)+f(v): u v \in E(G)\}$.

Clearly, $f$ is injective. To show that $f$ is an efficient zero ring labeling of $G$, we need to show that $|K|=\Delta(G)=1$ and $A_{0} \notin K$.

We obtain the sums

$$
f\left(w_{i-1}\right)+f\left(w_{2 m-i+1}\right)=A_{i-1}+A_{2 m-i+1}=A_{2 m}
$$


for $i=1,2, \ldots, m$. Then $K=\left\{A_{2 m}\right\}$ and thus $|K|=1$. Also, $A_{2 m} \neq A_{0}$ since $m>0$ and $m<m+p+1 \leq m+m+1=2 m+1$.

Case 2: The order of at least one caterpillar is at least three. Applying Lemma 1, we denote the vertices of each caterpillar such that the endvertices of the central path have no hanging leaf.

Let $G_{1}, G_{2}, \ldots, G_{p}$ denote the caterpillars. Suppose the length of the central path of $G_{i}$ has $s_{i}$ vertices. Denote the central path of $G_{1}$ by $\left[w_{1}, w_{2}, \ldots, w_{s_{1}}\right]$, and the central path of $G_{i}$ by $\left[w_{q+1}, w_{q+2}, \ldots, w_{q+s_{i}}\right]$ for $i=2,3, \ldots, p$, where $q=s_{1}+s_{2}+\cdots+s_{i-1}$. Suppose the number of hanging leaves of $w_{i}$ is $r_{i}$, and denote the hanging leaves of $w_{i}$ by $w_{i}^{j}$, where $j=1,2, \ldots, r_{i}$.

Suppose the maximum number of hanging leaves of a vertex in $G$ is $r_{k}$. Since the endvertices in the central path of each caterpillar in $G$ have no hanging leaf and there is at least one caterpillar with at least three vertices, it follows that $\Delta(G)=r_{k}+2$.

Consider a caterpillar $H$ such that $V(H)=V(G)$ and $E(H)=E(G) \cup\left\{\left\{w_{q_{i}}, w_{q_{i}}+1\right\}\right.$ : $i=1,2, \ldots p-1\}$, where $q_{i}=s_{1}+s_{2} \cdots+s_{i}$. By Theorem $6, H$ has an efficient zero ring labeling $h$.

Define a function $f: V(G) \rightarrow M_{2}^{0}\left(\mathbb{Z}_{n r_{k}+n-r_{k}}\right)$ such that $f(a)=h(a)$ for all $a \in$ $V(G)$. Since $h$ is injective, it follows that $f$ is also injective. $G$ is an edge-induced subgraph of $H$, so $A_{0} \notin K_{H}=\{h(u)+h(v): u v \in E(H)\}$ implies that $A_{0} \notin K=$ $\{f(u)+f(v): u v \in E(G)\}$. To show that $f$ is an efficient zero ring labeling of $G$, it remains to show that $|K|=\Delta(G)=r_{k}+2$.

Using the labeling in the proof of Theorem 4, we obtain

$$
K=\left\{A_{\left(r_{k}+1\right)(q-2)}, A_{\left(r_{k}+1\right)(q-2)+1}, \ldots, A_{\left(r_{k}+1\right)(q-2)+r_{k}}, A_{\left(r_{k}+1\right)(q-1)}\right\},
$$

where $q=s_{1}+s_{2}+\cdots+s_{p}$. Thus, $|K|=r_{k}+2=\Delta(G)$.

Example 13. Figure 14 shows an efficient zero ring labeling of a disjoint union of three caterpillars using $M_{2}^{0}\left(\mathbb{Z}_{66}\right)$. In this labeling, the set of sums is $K=\left\{A_{60}, A_{61}, A_{62}, A_{63}, A_{64}, A_{65}\right\}$ and thus $|K|=6$, which is the maximum degree of the graph.

Theorem 11. Let $G$ be a disjoint union of a finite number of lobsters in which each elbow has at most one hanging leaf. Then $G$ has an efficient zero ring labeling.

Proof. Let $G$ be a disjoint union of $p$ lobsters. We show that $G$ has an efficient zero ring labeling.

If the order of each lobster is one or two, then by Theorem 10, $G$ has an efficient zero ring labeling. Suppose the order of at least one lobster is at least three. Applying Lemma 2 , we denote the vertices of each lobster such that the endvertices of the central path have no hanging leaf.

Let $G_{1}, G_{2}, \ldots, G_{p}$ denote the disjoint lobster graphs. Suppose the length of the central path of $G_{i}$ has $s_{i}$ vertices. Denote the central path of $G_{1}$ by $\left[w_{1}, w_{2}, \ldots, w_{s_{1}}\right]$, and the central path of $G_{i}$ by $\left[w_{q+1}, w_{q+2}, \ldots, w_{q+s_{i}}\right]$ for $i=2,3, \ldots, p$, where $q=s_{1}+s_{2}+\cdots+s_{i-1}$. 


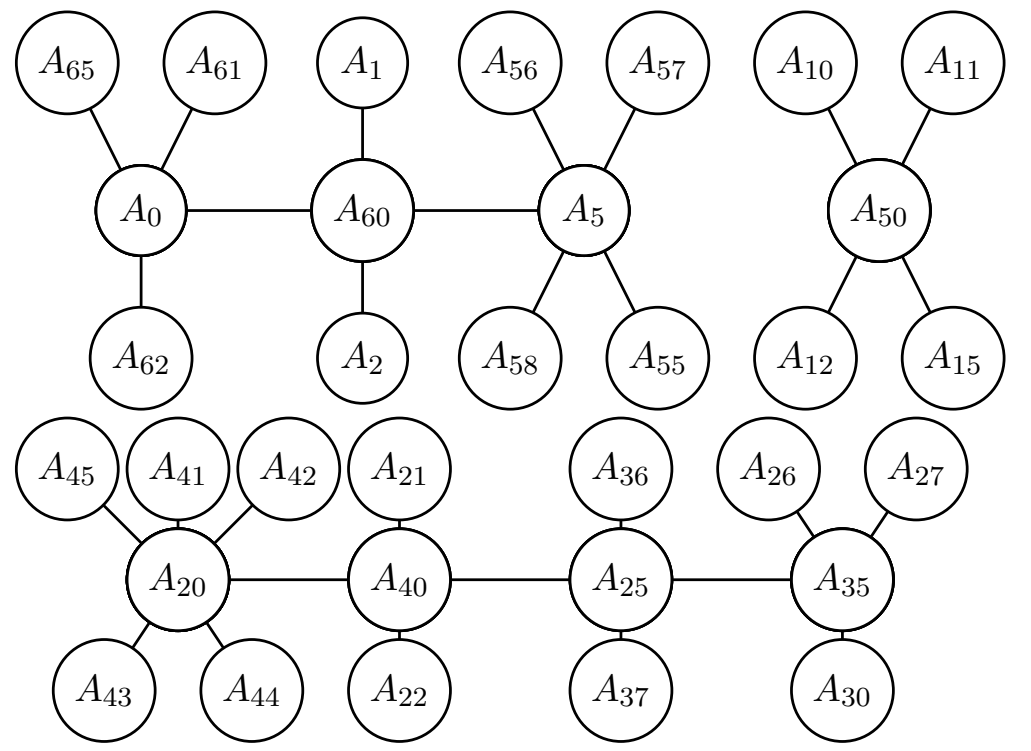

Figure 14: Efficient zero ring labeling of a disjoint union of three caterpillars using $M_{2}^{0}\left(\mathbb{Z}_{66}\right)$

Suppose the number of elbows of $w_{i}$ is $r_{i}$, and denote the elbows of $w_{i}$ by $w_{i, j}$, where $j=1,2, \ldots, r_{i}$. If it exists, denote the hanging leaf of $w_{i, j}$ by $w_{i, j, 1}$.

Suppose the maximum number of elbows of a vertex in $G$ is $r_{k}$. Since the endvertices in the central path of each lobster in $G$ have no elbow and there is at least one lobster with at least three vertices, it follows that $\Delta(G)=r_{k}+2$.

Consider a lobster $H$ such that $V(H)=V(G)$ and $E(H)=E(G) \cup\left\{\left\{w_{q_{i}}, w_{q_{i}}+1\right\}\right.$ : $i=1,2, \ldots p-1\}$, where $q_{i}=s_{1}+s_{2} \cdots+s_{i}$. By Theorem $8, H$ has an efficient zero ring labeling $h$.

Define a function $f: V(G) \rightarrow M_{2}^{0}\left(\mathbb{Z}_{2 n r_{k}+n-2 r_{k}}\right)$ such that $f(a)=h(a)$ for all $a \in$ $V(G)$. Since $h$ is injective, it follows that $f$ is also injective. $G$ is an edge-induced subgraph of $H$, so $A_{0} \notin K_{H}=\{h(u)+h(v): u v \in E(H)\}$ implies that $A_{0} \notin K=$ $\{f(u)+f(v): u v \in E(G)\}$. To show that $f$ is an efficient zero ring labeling of $G$, it remains to show that $|K|=\Delta(G)=r_{k}+2$.

Using the labeling in the proof of Theorem 8, we obtain

$$
K=\left\{A_{\left(2 r_{k}+1\right)(q-2)}, A_{\left(2 r_{k}+1\right)(q-2)+1}, \ldots, A_{\left(2 r_{k}+1\right)(q-2)+r_{k}}, A_{\left(2 r_{k}+1\right)(q-1)}\right\},
$$

where $q=s_{1}+s_{2}+\cdots+s_{p}$. Thus, $|K|=r_{k}+2=\Delta(G)$.

Example 14. Figure 15 shows an efficient zero ring labeling of a disjoint union of two lobsters using $M_{2}^{0}\left(\mathbb{Z}_{57}\right)$. In this labeling, the set of sums is $K=\left\{A_{49}, A_{50}, A_{51}, A_{52}, A_{56}\right\}$ and thus $|K|=5$, which is the maximum degree of the graph.

Theorem 12. A cycle graph with $n$ vertices has an efficient zero ring labeling if $n$ is even. 


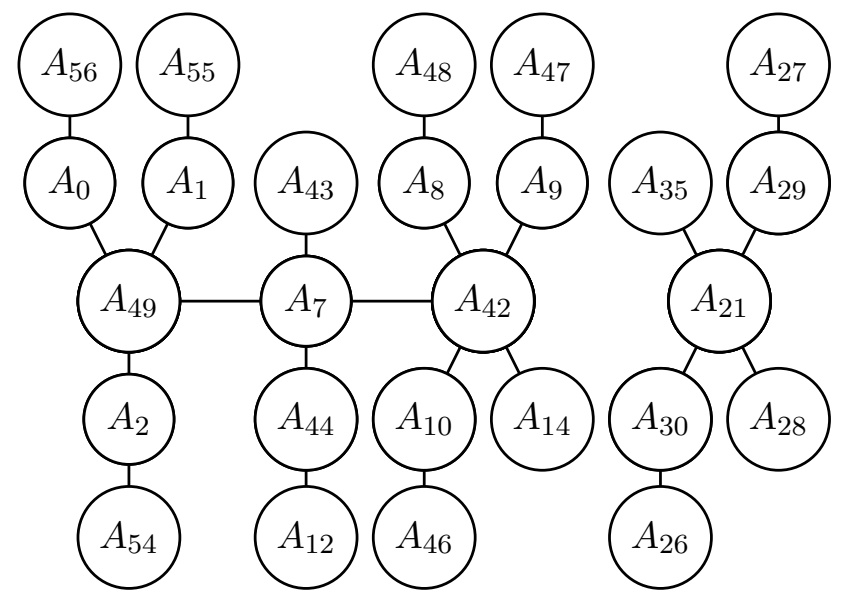

Figure 15: Efficient zero ring labeling of a disjoint union of two lobsters using $M_{2}^{0}\left(\mathbb{Z}_{57}\right)$

Proof. Let $C_{n}=\left[w_{1}, w_{2}, \ldots, w_{n}, w_{1}\right]$, where $n$ is even. Clearly, $\Delta\left(C_{n}\right)=2$. Define a function $f: V\left(C_{n}\right) \rightarrow M_{2}^{0}\left(\mathbb{Z}_{n}\right)$ such that

$$
f\left(w_{i}\right)=\left\{\begin{array}{ll}
A_{i-1} & \text { if } i \text { is odd } \\
A_{n-i+3} & \text { if } i \text { is even }
\end{array} .\right.
$$

Clearly, $f$ is injective. Let $K=\left\{f(u)+f(v): u v \in E\left(C_{n}\right)\right\}$. We obtain

$$
f\left(w_{i}\right)+f\left(w_{i+1}\right)=A_{i-1}+A_{n-(i+1)+3}=A_{n+1}=A_{1} \neq A_{0}
$$

if $i$ is odd, and

$$
f\left(w_{i}\right)+f\left(w_{i+1}\right)=A_{n-i+3}+A_{(i+1)-1}=A_{n+3}=A_{3} \neq A_{0}
$$

if $i$ is even. Thus, $K=\left\{A_{1}, A_{3}\right\}$, hence $|K|=2=\Delta\left(C_{n}\right)$. Therefore, $f$ is an efficient zero ring labeling of $C_{n}$.

Example 15. Figure 16 shows an efficient zero ring labeling of $C_{8}$ using $M_{2}^{0}\left(\mathbb{Z}_{8}\right)$. In this labeling, the set of sums is $K=\left\{A_{1}, A_{3}\right\}$ and thus $|K|=2=\Delta\left(C_{8}\right)$.

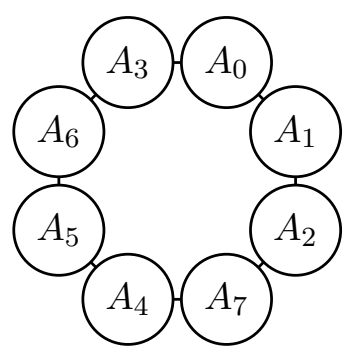

Figure 16: Efficient zero ring labeling of $C_{8}$ using $M_{2}^{0}\left(\mathbb{Z}_{8}\right)$ 
Theorem 13. A cycle graph with $n$ vertices has no efficient zero ring labeling if $n$ is odd.

Proof. Let $C_{n}=\left[w_{1}, w_{2}, \ldots, w_{n}, w_{1}\right]$, where $n$ is odd. Clearly, $\Delta\left(C_{n}\right)=2$. Assume that $C_{n}$ has an efficient zero ring labeling $f: V\left(C_{n}\right) \rightarrow R^{0}$.

Since $w_{1}$ and $w_{2}$ are adjacent, $f\left(w_{1}\right)+f\left(w_{2}\right) \in K=\left\{f(u)+f(v): u v \in E\left(C_{n}\right)\right\}$. It follows that $f\left(w_{2}\right)+f\left(w_{3}\right) \neq f\left(w_{1}\right)+f\left(w_{2}\right)$; otherwise, $f\left(w_{1}\right)=f\left(w_{3}\right)$, which is not possible since $f$ is injective. $K$ has only two elements, so $K=\left\{f\left(w_{1}\right)+f\left(w_{2}\right), f\left(w_{2}\right)+\right.$ $\left.f\left(w_{3}\right)\right\}$.

Similarly, $f\left(w_{3}\right)+f\left(w_{4}\right) \neq f\left(w_{2}\right)+f\left(w_{3}\right)$. Then $f\left(w_{3}\right)+f\left(w_{4}\right)=f\left(w_{1}\right)+f\left(w_{2}\right)$. Continuing in this manner, $f\left(w_{n}\right)+f\left(w_{1}\right)$ would be equal to $f\left(w_{1}\right)+f\left(w_{2}\right)$ since $n$ odd. But this implies that $f\left(w_{2}\right)=f\left(w_{n}\right)$. This is a contradiction since $f$ is injective.

Theorem 14. A complete bipartite graph has an efficient zero ring labeling.

Proof. Let $G=K_{m, n}$ be a complete bipartite graph with vertex set $\left\{x_{1}, x_{2}, \ldots, x_{m}\right\} \cup$ $\left\{y_{1}, y_{2}, \ldots, y_{n}\right\}$ and edge set $\left\{x_{i} y_{j}: 1 \leq i \leq m\right.$ and $\left.1 \leq j \leq n\right\}$. Without loss of generality, suppose $m \geq n$. Then $\Delta(G)=m$. Define a function $f: V(G) \rightarrow M_{2}^{0}\left(\mathbb{Z}_{2 m}\right)$ such that $f\left(x_{i}\right)=A_{2 i-2}$ and $f\left(y_{j}\right)=A_{2 j-1}$.

Clearly, $f$ is injective. Let $K=\{f(u)+f(v): u v \in E(G)\}$. We obtain

$$
f\left(x_{i}\right)+f\left(y_{j}\right)=A_{2 i-2}+A_{2 j-1}=A_{2 i+2 j-3}
$$

for $i=1,2, \ldots, m$ and $j=1,2, \ldots, n$. Then $K=\left\{A_{1}, A_{3}, \ldots, A_{2 m-1}\right\}$, hence $|K|=m$. Moreover, since $m$ is at least one, $A_{0} \notin K$. Thus, $f$ is an efficient zero ring labeling of $G$.

Example 16. Figure 17 shows an efficient zero ring labeling of $K_{5,4}$ using $M_{2}^{0}\left(\mathbb{Z}_{10}\right)$. In this labeling, the set of sums is $K=\left\{A_{1}, A_{3}, A_{5}, A_{7}, A_{9}\right\}$ and thus $|K|=5=\Delta\left(K_{5,4}\right)$.

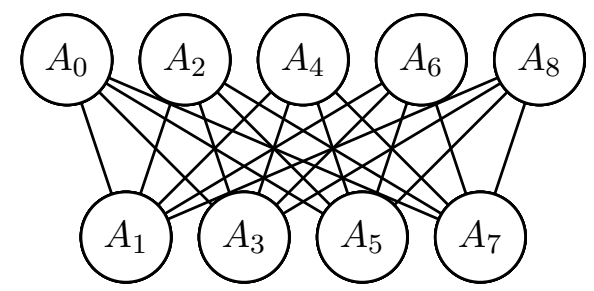

Figure 17: Efficient zero ring labeling of $K_{5,4}$ using $M_{2}^{0}\left(\mathbb{Z}_{10}\right)$

Theorem 15. A fan graph $F_{n}$ has an efficient zero ring labeling for $n \geq 3$.

Proof. It was shown in [2] that $F_{n}$ has an optimal zero ring labeling for $n \geq 3$. By definition of $F_{n}$, it has a vertex with degree $\left|F_{n}\right|-1$. Thus, by Theorem $3, F_{n}$ has an efficient zero ring labeling for $n \geq 3$.

Theorem 16. A wheel graph $W_{n}$ has an efficient zero ring labeling for $n \geq 3$. 
Proof. It was shown in [2] that $W_{n}$ has an optimal zero ring labeling for $n \geq 3$. By definition of $W_{n}$, it has a vertex with degree $\left|W_{n}\right|-1$. Thus, by Theorem $3, W_{n}$ has an efficient zero ring labeling for $n \geq 3$.

Theorem 17. A friendship graph $T_{n}$ has an efficient zero ring labeling for $n \geq 2$.

Proof. It was shown in [2] that $T_{n}$ has an optimal zero ring labeling for $n \geq 2$. By definition of $T_{n}$, it has a vertex with degree $\left|T_{n}\right|-1$. Thus, by Theorem $3, T_{n}$ has an efficient zero ring labeling for $n \geq 2$.

Example 17. Figure 18 shows an efficient zero ring labeling of $F_{5}$ using $M_{2}^{0}\left(\mathbb{Z}_{6}\right)$. Figure 19 shows an efficient zero ring labeling of $W_{8}$ using $M_{2}^{0}\left(\mathbb{Z}_{8}\right)$. Figure 20 shows an efficient zero ring labeling of $T_{4}$ using $M_{2}^{0}\left(\mathbb{Z}_{9}\right)$. In these labelings, the set of sums is the difference of the zero ring used and $\left\{A_{0}\right\}$.

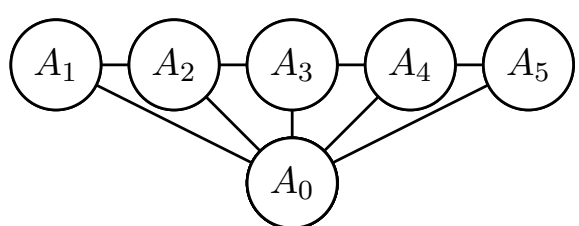

Figure 18: Efficient zero ring labeling of $F_{5}$ using $M_{2}^{0}\left(\mathbb{Z}_{6}\right)$

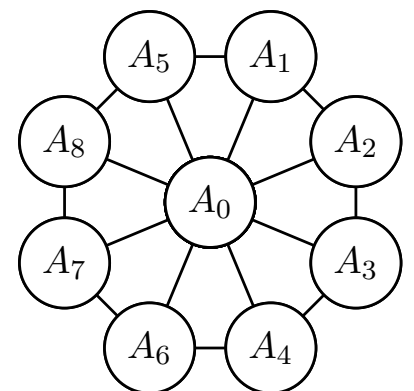

Figure 19: Efficient zero ring labeling of $W_{8}$ using $M_{2}^{0}\left(\mathbb{Z}_{9}\right)$

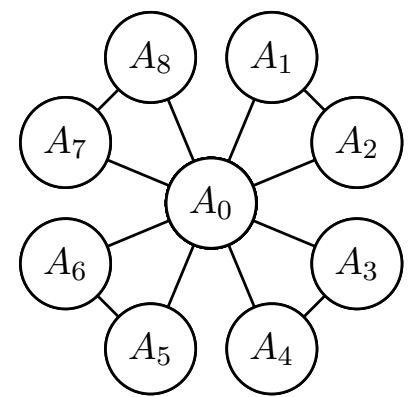

Figure 20: Efficient zero ring labeling of $T_{4}$ using $M_{2}^{0}\left(\mathbb{Z}_{9}\right)$

\section{Acknowledgements}

The authors are grateful to the blind peer reviewers who helped improve the paper, as well as Dr. Severino V. Gervacio, Dr. Leonor A. Ruivivar, and Dr. Neil M. Mame who contributed invaluable comments and suggestions over the course of this study. 
This work was funded by the Department of Science and Technology-Science Education Institute (DOST-SEI) and the Commission on Higher Education (CHED) of the Philippine government.

\section{References}

[1] Mukti Acharya, Pranjali, and Purnima Gupta. Zero ring labeling of graphs. Electronic Notes in Discrete Mathematics, 48:65-72, July 2015.

[2] Michelle Dela Rosa-Reynera. On Graphs of Minimum Zero Ring Index. PhD thesis, August 2018.

[3] Reinhard Diestel. Graph Theory. Springer, 4th edition, 2010.

[4] Joseph A. Gallian. Contemporary Abstract Algebra. Brooks/Cole, 8th edition, 2013.

[5] Frank Harary. Graph Theory. Addison-Wesley, 1969.

[6] Pranjali, Mukti Acharya, and Purnima Gupta. Further results on zero ring labeling of graphs. Bulletin of the International Mathematical Virtual Institute, 5:205-210, 2014. 